\title{
Designing student projects for teaching and learning discrete mathematics and computer science via primary historical sources ${ }^{* \dagger}$
}

\author{
Janet Heine Barnett \\ Dept. of Mathematics and Physics \\ Colorado State University - Pueblo \\ Pueblo, CO 81001, USA \\ janet.barnett@colostate-pueblo.edu \\ Jerry Lodder, David Pengelley \\ Dept. of Mathematical Sciences \\ New Mexico State University \\ Las Cruces, NM 88003, USA \\ jlodder@nmsu.edu, davidp@nmsu.edu \\ Inna Pivkina, Desh Ranjan \\ Dept. of Computer Science \\ New Mexico State University \\ Las Cruces, NM 88003, USA \\ inna@nmsu.edu,dranjan@nmsu.edu
}

October 10, 2009

\footnotetext{
Abstract

${ }^{*}$ Based on a workshop presented at the HPM Group Satellite Meeting of ICME 11 (HPM 2008) in Mexico City, Mexico, 14-18 July 2008.

$\dagger$ Keywords: discrete mathematics, graph theory, combinatorics, logic, computer science, pedagogy, motivation, primary historical sources, student projects.
} 
We discuss and present excerpts from student projects being developed and tested by an interdisciplinary faculty team for courses in discrete mathematics, graph theory, combinatorics, logic, and computer science. The goal of our work is to provide motivation, direction, and context for these subjects through student projects based directly on the writings of the pioneers who first developed crucial ideas and worked on seminal problems. Each project is built around primary source material close to or representing the discovery of a key concept. Through guided reading and activities, students explore the mathematics of the original discovery and develop their own understanding of the subject. We illustrate how project design elements support our pedagogical goals and discuss classroom implementation. Further evaluation and project development is underway, and two web sites provide expanded materials and information. Ongoing support is provided by the US National Science Foundation.

\section{Introduction}

A discrete mathematics course often teaches about precise logical and algorithmic thought and methods of proof to students studying mathematics, computer science, or teacher education. The roots of such methods of thought, and of discrete mathematics itself, are as old as mathematics, with the notion of counting, a discrete operation, usually cited as the first mathematical development in ancient cultures (Katz 1998). However, a typical course frequently presents a fast-paced news reel of facts and formulae, often memorized by the students, with the text offering only passing mention of the motivating problems and original work that eventually found resolution in the modern concepts. This paper describes a pedagogical approach to teaching topics in discrete mathematics and computer science intended to place the material in context and provide direction to the subject matter via student projects centered around actual excerpts from primary historical sources. Much has already been written about teaching with primary historical sources (Fauvel \& van Maanen, 2000, ch. 9). Here we focus on a list of specific pedagogical goals and how they can be achieved through design of student projects based on primary sources.

Our interdisciplinary team of mathematics and computer science faculty has completed a pilot program funded by the US National Science Foundation, in which we have developed and tested over a dozen historical projects 
for student work in courses in discrete mathematics, graph theory, combinatorics, logic, and computer science. These projects have appeared in print (Barnett et al 2009), and are presently available through the web resource (Bezhanishvili et al 2003).

We are now in the second year of a four-year NSF expansion grant through which additional projects based on primary sources are being developed, tested, evaluated, revised, and published. The expansion will support classroom testing by faculty at twenty other institutions, careful evaluation of their effectiveness, and provide training in teaching with these projects to graduate students. Projects created to date under the expansion grant are available at our new web resource (Barnett et al 2008), along with descriptions of projects yet to be developed. We welcome instructors who would like to collaborate in testing or writing projects.

Here we will briefly describe the pedagogical goals which guide project design, illustrate these principles with excerpts from two particular projects, discuss how such historical projects can be implemented in the classroom, and present preliminary evaluation evidence of their effectiveness.

\section{Pedagogical Goals and Design Principles}

A central goal of our pedagogical approach is to recover motivation for studying particular core topics by teaching and learning these topics directly from a primary historical source of scientific significance. Primary source authors represented in the collection include Archimedes, Cantor, Euler, Hamilton, Leibniz, Pascal, Shannon, Turing, Veblen, and von Neumann, writing on topics such as mathematical induction, finite sums of powers, graph theory, transfinite arithmetic, binary arithmetic, combinatorics, computability, and decidability.

Designed to capture the spark of discovery and motivate subsequent lines of inquiry, each historical project is built around primary source material which serves either as an introduction to a core topic in the curriculum, or as supplementary material to a textbook treatment of that topic. Through guided reading of the selected primary source material and by completing a sequence of activities based on these excerpts, students explore the science of the original discovery and develop their own understanding of the subject. Each project also provides a discussion of the historical exigency of the piece and a few biographical comments about the author to place the source in 
context.

The following pedagogical goals further guide our selection of primary source material and the design of a project. The next section will illustrate how these goals have been implemented in two particular projects.

\section{Fifteen Pedagogical Goals Guiding the Development of Primary Source Based Projects}

1. Hone students' verbal and deductive skills through reading.

2. Provide practice moving from verbal descriptions of problems to precise mathematical formulations.

3. Promote recognition of the organizing concept behind a procedure.

4. Promote understanding of the present-day paradigm of the subject through the reading of an historical source which requires no knowledge of that paradigm.

5. Promote reflection on present-day standards and paradigm of subject.

6. Draw attention to subtleties, which modern texts may take for granted, through the reading of an historical source.

7. Promote students' ability to equally participate, regardless of their background or capability.

8. Offer diverse approaches to material which can serve to benefit students with different learning styles through exposure to multiple approaches.

9. Provide a point of departure for students' work, and a direction for their efforts.

10. Encourage more authentic (versus routine) student proof efforts through exposure to original problems in which the concepts arose.

11. Promote a human vision of science and of mathematics.

12. Provide a framework for the subject in which all elements appear in their right place.

13. Promote a dynamical vision of the evolution of mathematics. 
14. Promote enriched understanding of subject through greater understanding of its roots, for students and instructors.

15. Engender cognitive dissonance (dépaysement) when comparing a historical source with a modern textbook approach, which to resolve requires an understanding of both the underlying concepts and use of present-day notation.

\section{Incorporating pedagogical design goals: two sample projects}

In this section we provide excerpts from two student projects in order to give a flavor of their nature. For each, we display selected excerpts from the primary historical source in the project, from our own narrative in the project, and also from student assignment questions, and discuss how these elements promote our pedagogical goals. To set project excerpts apart from our writing in this paper, all project excerpts are indented relative to our main text and bracketed by the symbol

\$ONO0000000

at their beginning and end. Moreover, note that within the project excerpts, the primary historical source material is set in sans serif font. Our specific pedagogical goals are referred to by the numbers used to enumerate them in Section 2.

\subsection{Treatise on the Arithmetical Triangle: Blaise Pas- cal}

The project Treatise on the Arithmetical Triangle is intended for an introductory level discrete mathematics and proofs course, and presents the concept of mathematical induction from the pioneering work written by Blaise Pascal (Pascal 1991) in the 1650s.

After arranging the figurate numbers in a table, forming "Pascal's triangle," Pascal observes several patterns in the table, which he would like to claim continue indefinitely. Pascal offers a condition for the persistence of a 
pattern, stated verbally in the proof of his "Twelfth Consequence," a condition known today as mathematical induction. In fact, Pascal's treatise is the first place in the mathematical literature where the principle of mathematical induction is enunciated so completely and generally (Goals 11,12,13,14,15). Moreover, Pascal's Twelfth Consequence results directly in the modern factorial formula for combination numbers and binomial coefficients, allowing him to proceed to algebra and probability. In this project, students learn firsthand about the issues involved in proofs by iteration, generalizable example, and mathematical induction.

Students begin by reading Pascal's defining description of his triangle, which is highly verbal, entirely labeled using letters, and rotated in comparison to how most people view it today. We display excerpts from a few of his critical definitions here. Such a verbal approach, to a triangle all students think they are already familiar with, but without modern indexing notation, and geometrically tilted from the modern view, challenges students' skill at translating to modern mathematical descriptions, and places all students on the same unfamiliar footing (Goals 1,2,7,15).

The idea of having students learn by being placed on unfamiliar footing is expressed in French as dépaysement, a condition in which one must approach things from unaccustomed points of view, and pay great attention to subtleties (Goals 3,4,5,6,8,15). One might translate it as 'being thrown off guard', 'being bewildered', 'being taken out of one's element'. It is one of the great strengths of learning from primary historical sources, since they were often written in dramatically different context and time, thus providing a very distinct, and often extremely valuable, point of view. By viewing a topic from a point of view different from the standard modern one, a broader, but also deeper and more flexible, understanding is gained (Goal 14).

100000000000

TREATISE ON THE ARITHMETICAL TRIANGLE

\section{DEFINITIONS}

... Through each of the points of section and parallel to the sides I draw lines whose intersections make little squares which I call cells. 


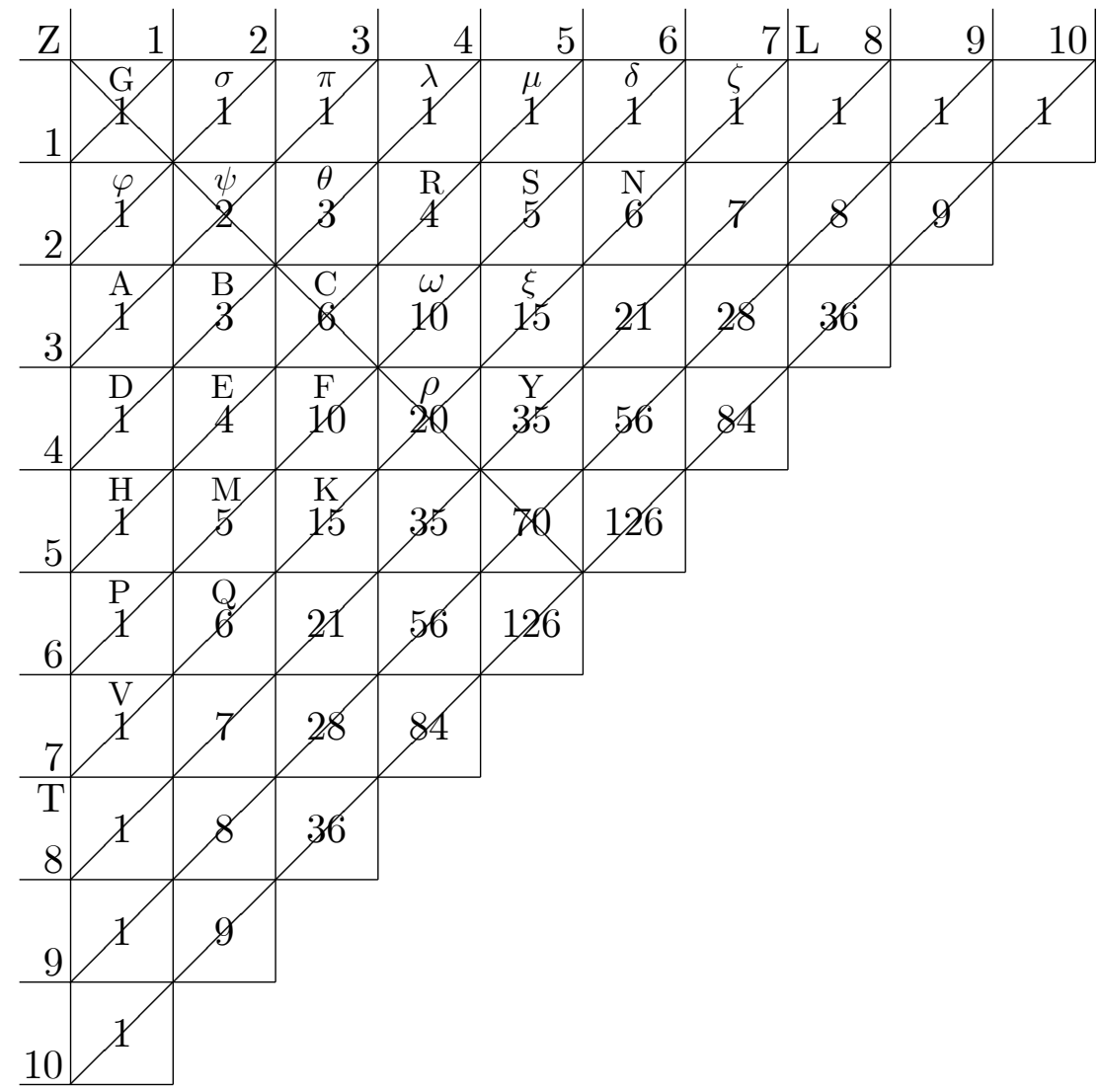

Cells between two parallels drawn from left to right are called cells of the same parallel row, as, for example, cells $G, \sigma, \pi$, etc., or $\varphi$, $\psi, \theta$, etc.

Those between two lines drawn from top to bottom are called cells of the same perpendicular row, as, for example, cells $G, \varphi, A, D$, etc., or $\sigma, \psi, B$, etc.

Those cut diagonally by the same base are called cells of the same base, as, for example, $D, B, \theta, \lambda$, or $A, \psi, \pi$. . .

Cells of the same base equidistant from its extremities are called reciprocals, as, for example, $E, R$ and $B, \theta, \ldots$

Now the numbers assigned to each cell are found by the following method: 
The number of the first cell, which is at the right angle, is arbitrary; ... Each of the others is specified by a single rule as follows:

The number of each cell is equal to the sum of the numbers of the perpendicular and parallel cells immediately preceding. Thus cell $F$, that is, the number of cell $F$, equals the sum of cell $C$ and cell $E$, and similarly with the rest.

\section{COCOCOCOCOOO}

Formalizing Pascal's entire description using modern indexing notation is the first big challenge to students, through exercises like the following (Goals 1,2,4,5). Sophisticated ideas like double-indexing arise immediately and naturally (Goal 6), and students will also learn from this project, in relevant context, about summation and product notations, and recurrence relations (Goals 4,5,8).

\section{DOCONOCONOCO}

1. Pascal's Triangle and its numbers

(a) Let us use the notation $T_{i, j}$ to denote what Pascal calls the number assigned to the cell in parallel row $i$ (which we today call just row $i$ ) and perpendicular row $j$ (which we today call column $j$ ). We call the $i$ and $j$ by the name indices (plural of index) in our notation. Using this notation, explain exactly what Pascal's rule is for determining all the numbers in all the cells. Be sure to give full details. This should include explaining for exactly which values of the indices he defines the numbers.

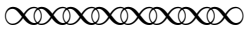

After Pascal proves some simple properties of the triangle essentially iteratively, mathematical induction first arises naturally but implicitly in demonstrating its symmetry. In addition, Pascal proves his claim by "generalizable example," largely because he has no indexing notation to deal conveniently with arbitrary elements. Having students make all this precise in full generality with modern notation enables them to begin to think in terms of induction before it is formally introduced, and to powerfully appreciate the efficacy of indexing notation (Goals $3,4,5,10$ ). 
corococosococos

\section{Fifth Consequence}

In every arithmetical triangle each cell is equal to its reciprocal.

For in the second base, $\phi \sigma$, it is evident that the two reciprocal cells, $\varphi, \sigma$, are equal to each other and to $G$.

In the third base, $A, \psi, \pi$, it is also obvious that the reciprocals, $\pi, A$, are equal to each other and to $G$.

In the fourth base it is obvious that the extremes, $D, \lambda$, are again equal to each other and to $G$.

And those between, $B, \theta$, are obviously equal since $B=$ $A+\psi$ and $\theta=\pi+\psi$. But $\pi+\psi=A+\psi$ by what has just been shown. Therefore, etc.

Similarly it can be shown for all the other bases that reciprocals are equal, because the extremes are always equal to $G$ and the rest can always be considered as the sum of cells in the preceding base which are themselves reciprocals.

3. Symmetry in the triangle: first contact with mathematical induction

Write the Fifth Consequence using our index notation. Use index notation and the ideas in Pascal's proof to prove the Consequence in full generality, not just for the example he gives. Explain the conceptual ideas behind the general proof.

\section{0}

Only after students grapple with and explain Pascal's Fifth Consquence in their own way are they expected to read about the principle of mathematical induction. The crowning consequence in Pascal's treatise is the Twelfth, in which Pascal derives a formula for the ratio of consecutive numbers along a base in the triangle. From this he will obtain an elegant and efficient "closed" formula for all the numbers in the triangle, a powerful tool for much future mathematical work. And it is right here that Pascal enunciates the general 
proof principle we call induction. Again we ask students to translate Pascal's proof by generalizable example into a modern and completely general proof. This is far from trivial, and even involves an understanding of a property of proportions that is largely lost today. (This single rich source excerpt and its tasks for students encompasses all fifteen of our pedagogical goals.)

corococosocos

\section{Twelfth Consequence}

In every arithmetical triangle, of two contiguous cells in the same base the upper is to the lower as the number of cells from the upper to the top of the base is to the number of cells from the lower to the bottom of the base, inclusive.

Let any two contiguous cells of the same base, $E, C$, be taken. I say that

\begin{tabular}{|c|c|c|c|}
\hline $\begin{array}{c}E \\
\text { the } \\
\text { lower }\end{array}$ & $\begin{array}{c}C \\
\text { the } \\
\text { upper }\end{array}$ & \begin{tabular}{l}
\multicolumn{1}{c}{2} \\
because there \\
are two cells \\
from $E$ to the \\
bottom, namely \\
$E, H$,
\end{tabular} & $\begin{array}{l}\quad 3 \\
\text { because there } \\
\text { are three cells } \\
\text { from } C \text { to the } \\
\text { top, namely } \\
C, R, \mu \text {. }\end{array}$ \\
\hline
\end{tabular}

Although this proposition has an infinity of cases, I shall demonstrate it very briefly by supposing two lemmas:

The first, which is self-evident, that this proportion is found in the second base, for it is perfectly obvious that $\varphi: \sigma:: 1: 1$;

The second, that if this proportion is found in any base, it will necessarily be found in the following base.

Whence it is apparent that it is necessarily in all the bases. For it is in the second base by the first lemma; therefore by the second lemma it is in the third base, therefore in the fourth, and to infinity. 
It is only necessary therefore to demonstrate the second lemma as follows: If this proportion is found in any base, as, for example, in the fourth, $D \lambda$, that is, if $D: B:: 1: 3$, and $B: \theta:: 2: 2$, and $\theta: \lambda:: 3: 1$, etc., I say the same proportion will be found in the following base, $H \mu$, and that, for example, $E: C:: 2: 3$.

For $D: B:: 1: 3$, by hypothesis.

\begin{tabular}{|c|c|}
\hline Therefore & $D+B$ \\
\hline
\end{tabular}

Similarly $B: \theta:: 2: 2$, by hypothesis

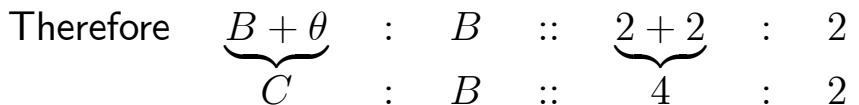

But $B \quad: \quad E \quad:: \quad 3 \quad 3 \quad: 4$

Therefore, by compounding the ratios, $C: E:: 3$ :

2 .

Q.E.D.

The proof is the same for all other bases, since it requires only that the proportion be found in the preceding base, and that each cell be equal to the cell before it together with the cell above it, which is everywhere the case.

6. Pascal's Twelfth Consequence: the key to our modern factorial formula

(a) Rewrite Pascal's Twelfth Consequence as a generalized modern formula, entirely in our $T_{i, j}$ terminology. Also verify its correctness in a couple of examples taken from his table in the initial definitions section.

(b) Adapt Pascal's proof by example of his Twelfth Consequence into modern generalized form to prove the formula you obtained above. Use the principle of mathematical induction to create your proof.

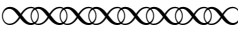

From his Twelfth Consequence Pascal can develop a "formula" for the numbers in the triangle, which can then be used in future work on combinatorics, probability, and algebra. We have students follow Pascal's generalizable example to do so (Goals $1,2,5,8,9,14,15$ ) in modern form. 
10000000000

PROBLEM

Given the perpendicular and parallel exponents of a cell, to find its number without making use of the arithmetical triangle. ...

7. Pascal's formula for the numbers in the Arithmetical Triangle

(a) Write down the general formula Pascal claims in solving his "Problem." Your formula should read $T_{i, j}=$ "some formula in terms of $i$ and $j$." Also write your formula entirely in terms of factorials.

(b) Look at the reason Pascal indicates for his formula for a cell, and use it to make a general proof for your formula above for an arbitrary $T_{i, j}$. You may try to make your proof just like Pascal is indicating, or you may prove it by mathematical induction.

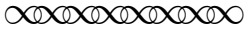

The project can continue on perfectly naturally to integrate combinatorics, the binomial theorem, Fermat's Theorem (proof by induction on the base using Pascal's formula for the binomial coefficients and uniqueness of prime factorization), and end with the RSA cryptosystem. This goes far beyond the historical source, but shows how the source serves as foundation for important modern topics (Goals 9,14).

\subsection{The solution of a problem relating to the geometry of position: Leonhard Euler}

The project Treatise on the Arithmetical Triangle just discussed illustrates how rich individual small excerpts from a primary source can be in terms of promoting the pedagogical goals of our approach to using history in discrete mathematics courses. Next we illustrate how specific pedagogical goals can be emphasized in a project by using the project narrative and student tasks to 
carefully frame a sequence of excerpts from a primary source. The project we consider, Early Writings on Graph Theory: Euler Circuits and The Königsberg Bridge Problem, is suitable for a beginning-level discrete mathematics course, or for a 'transition to proof' course.

In the paper (Euler 1758-59) on which this project is based, Leonhard Euler (1707-1783) provides a mathematical formulation of the Königsberg Bridge Problem. Considered today to be the starting point of modern graph theory, this foundational paper offers a unique window on a dynamical vision of the evolution of mathematics (Goal 13). The first Euler excerpt which students read in the project conveys this vision in his own words.

00000000000

SOLUTIO PROBLEMATIS AD GEOMETRIAM SITUS PERTINENTIS

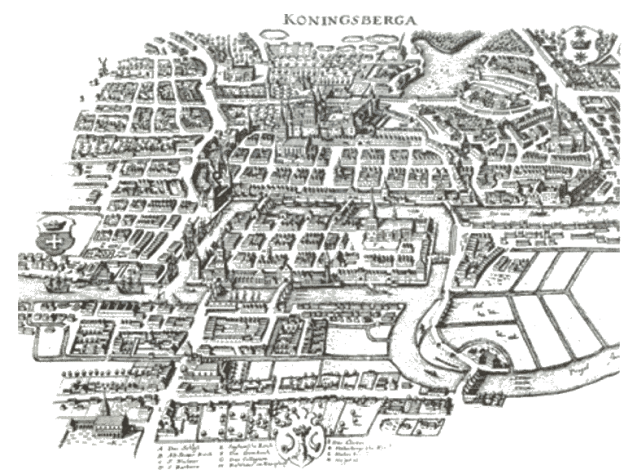

FIG. 1.1

1 In addition to that branch of geometry which is concerned with magnitudes, and which has always received the greatest attention, there is another branch, previously almost unknown, which Leibniz first mentioned, calling it the geometry of position. This branch is concerned only with the determination of position and its properties; it does not involve measurements, nor calculations made with them. It has not yet been satisfactorily determined what kind of problems are relevant to this geometry of position, or what methods should be used in solving them. Hence, when a problem was recently mentioned, which 
seemed geometrical but was so constructed that it did not require the measurement of distances, nor did calculation help at all, I had no doubt that it was concerned with the geometry of position - especially as its solution involved only position, and no calculation was of any use. I have therefore decided to give here the method which I have found for solving this kind of problem, as an example of the geometry of position.

\section{0}

The project places further emphasis on this evolutionary vision of mathematics in two ways. First, the project's introduction places Euler's paper in a historical context with a first-hand account ${ }^{1}$ from Gottfried W. Leibniz (1646 - 1716) concerning why he felt the need for a 'geometry of position,' followed by a later account ${ }^{2}$ of the state of this new field in 1833 from C. F. Gauss (1777 - 1855). Secondly, the project explicitly introduces the more abstract terminology and notation of modern graph theory in parallel with Euler's analysis, as illustrated in the following project excerpt.

\section{0}

2 The problem, which I am told is widely known, is as follows: in Königsberg in Prussia, there is an island $A$, called the Kneiphof; the river which surrounds it is divided into two branches, as can be seen in Fig. [1.2], and these branches are crossed by seven bridges, $a, b, c, d, e, f$ and $g$. Concerning these bridges, it was asked whether anyone could arrange a route in such a way that he would cross each bridge once and only once. I was told that some people asserted that this was impossible,

\footnotetext{
${ }^{1}$ This appeared in an 1670 letter from Leibniz to Christian Huygens (1629 - 1695), quoted in (Biggs \& Wilson 1976, p. 30) as follows: 'I am not content with algebra, in that it yields neither the shortest proofs nor the most beautiful constructions of geometry. Consequently, in view of this, I consider that we need yet another kind of analysis, geometric or linear, which deals directly with position, as algebra deals with magnitude.'

${ }^{2}$ As quoted in (Biggs \& Wilson 1976, p. 30), Gauss reported 'Of the geometry of position, which Leibniz initiated and to which only two geometers, Euler and Vandermonde, have given a feeble glance, we know and possess, after a century and a half, very little more than nothing.'
} 
while others were in doubt: but nobody would actually assert that it could be done. From this, I have formulated the general problem: whatever be the arrangement and division of the river into branches, and however many bridges there be, can one find out whether or not it is possible to cross each bridge exactly once?

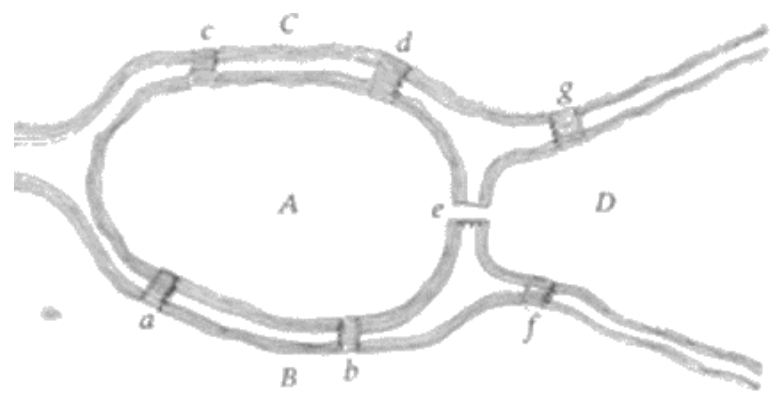

FIG. 1.2

Notice that Euler begins his analysis of the 'bridge crossing' problem by first replacing the map of the city by a simpler diagram showing only the main feature. In modern graph theory, we simplify this diagram even further to include only points (representing land masses) and line segments (representing bridges). These points and line segments are referred to as vertices (singular: vertex) and edges respectively. The collection of vertices and edges together with the relationships between them is called a graph. More precisely, a graph consists of a set of vertices and a set of edges, where each edge may be viewed as an ordered pair of two (usually distinct) vertices. In the case where an edge connects a vertex to itself, we refer to that edge as a loop.

1. Sketch the diagram of a graph with 5 vertices and 8 edges to represent the following bridge problem. 


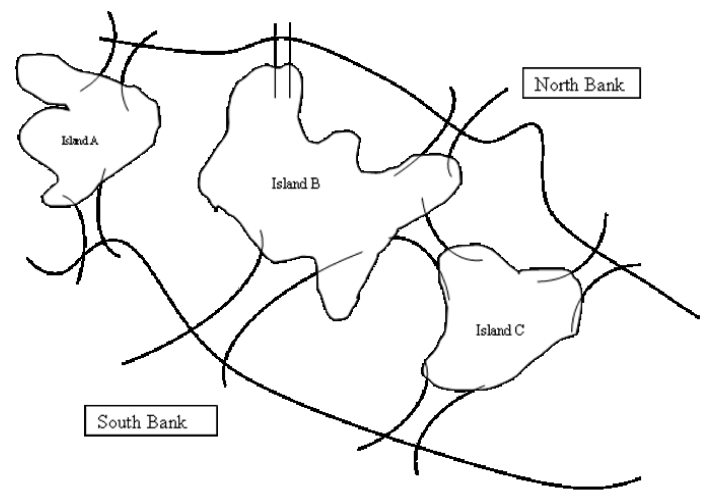

10000000000

Through this interweaving of primary source excerpts, project narrative and project questions, students are thus prompted to connect Euler's writing with the modern theory which (eventually) evolved out of it from the start of the project. Additional definitions are introduced and analyzed as they arise in connection with Euler's analysis of the bridge crossing problem. This strategy is also one way to promote the pedagogical goal of building students' understanding of the subject in its current form without requiring prior knowledge of its present-day paradigm to read the historical sources (Goal 4). In the Arithmetical Triangle project, this is done by referring students to a current textbook at appropriate points; other projects promote this goal through the use of concluding sections which connect the mathematical concepts developed in the primary source to its current terminology and notation. ${ }^{3}$

The strategy employed in the Euler Circuits project of developing the modern theory in parallel with its original formulation also enhances students' ability to more equally participate, regardless of their background or capability (Goal 7). This goal is further advanced by the primary source selections themselves. For example, Euler develops two procedures in his paper for determining whether a given bridge crossing problem admits of a solution, the first of which is quite distinct from the usual process presented in a current textbook for determining whether a given graph contains an Euler

\footnotetext{
${ }^{3}$ See, for example, Origins of Boolean Algebra in the Logic of Classes: George Boole, John Venn and C. S. Peirce, author Janet Heine Barnett, available on the web resource (Barnett et al 2008).
} 
path. ${ }^{4}$ Thus, even students who have studied graph theory in another course are exposed to an algorithm which is not only different from the one they are likely to have encountered previously, but also considerably more concrete. The concrete nature of Euler's preliminary algorithm has further advantages, including that of benefiting students with different learning styles through exposure to multiple approaches (Goal 8).

Euler's derivation of his first algorithm for determining if a bridge crossing problem is solvable (i.e., if a graph contains an Euler path) occupies several pages in his paper, following which he gives two examples of its use. He then states the following two observations which he will use to develop his second (simpler) procedure.

\section{0}

16 In this way it will be easy, even in the most complicated cases, to determine whether or not a journey can be made crossing each bridge once and once only. I shall, however, describe a much simpler method for determining this which is not difficult to derive from the present method, after I have first made a few preliminary observations. First, I observe that the numbers of bridges written next to the letters $A, B, C$, etc. together add up to twice the total number of bridges. The reason for this is that, in the calculation where every bridge leading to a given area is counted, each bridge is counted twice, once for each of the two areas which it joins.

17 It follows that the total of the numbers of bridges leading to each area must be an even number, since half of it is equal to the number of bridges. This is impossible if only one of these numbers is odd, or if three are odd, or five, and so on. Hence if some of the numbers of bridges attached to the letters A, B, C, etc. are odd, then there must be an even number of these. Thus, in the Königsberg problem, there were odd numbers attached to the letters $A, B, C$ and $D$, as can be seen from Paragraph 14, and in the last example (in Paragraph 15),

\footnotetext{
${ }^{4}$ As we will see below, the second procedure which Euler develops and justifies is the more streamlined procedure of checking the parity of the vertex degrees which is standard in current textbook treatments.
} 
only two numbers were odd, namely those attached to $D$ and E.

\section{0}

As students work through Euler's various arguments, project questions such as the following also prompt them to pay attention to his style of argumentation, and to reflect upon how it differs from that used in a modern textbook, again emphasizing an evolutionary vision of mathematics while engendering cognitive dissonance through the comparison of a historical source with a modern textbook approach (Goals 13, 15).

10000000000

6. The result described in Paragraph 16 is sometimes referred to as 'The Handshake Theorem,' based on the equivalent problem of counting the number of handshakes that occur during a social gathering at which every person present shakes hands with every other person present exactly once. A modern statement of the Handshake Theorem would be: The sum of the degree of all vertices in a finite graph equals twice the number of edges in the graph. Locate this theorem in a modern textbook, and comment on how the proof given there compares to Euler's discussion in paragraph 16.

7. The result described in Paragraph 17 can be re-stated as follows: Every finite graph contains an even number of vertices with odd degree. Locate this theorem in a modern textbook, and comment on how the proof given there compares to Euler's discussion in paragraph 17.

\section{0}

The theme of reflection on present-day proof standards (Goal 5) apparent in these questions is also raised in the introduction of the project, where the computer-assisted resolution of the Four Color Problem by Appel and Haken is discussed. The project's emphasis on this theme culminates with a set of final exercises in which students are asked to 'fill in the gaps' of a 
modern proof of Euler's final theorem. Students first read Euler's own proof of this theorem, which appears in the paragraphs leading up to its statement. The following project excerpt gives his statement of the theorem, along with portions of the associated project narrative and exercises that continue the theme of reflection on present-day standards, while also drawing students' attention to subtleties (e.g., connectedness) which modern texts often take for granted (Goal 6).

100000000000

20 So whatever arrangement may be proposed, one can easily determine whether or not a journey can be made, crossing each bridge once, by the following rules:

If there are more than two areas to which an odd number of bridges lead, then such a journey is impossible.

If, however, the number of bridges is odd for exactly two areas, then the journey is possible if it starts in either of these areas.

If, finally, there are no areas to which an odd number of bridges leads, then the required journey can be accomplished starting from any area.

With these rules, the given problem can always be solved.

A complete modern statement of Euler's main result requires one final definition: a graph is said to be connected if for every pair of vertices $u, v$ in the graph, there is a walk from $u$ to $v$. Notice that a graph which is not connected will consist of several components, or subgraphs, each of which is connected. With this definition in hand, the main results of Euler's paper can be stated as follows:

Theorem: A finite graph $G$ contains an Euler circuit if and only if $G$ is connected and contains no vertices of odd degree. 
Corollary: A finite graph $G$ contains an Euler path if and only if $G$ is connected and contains at most two vertices of odd degree.

8. Illustrate why the modern statement specifies that $G$ is connected by giving an example of a disconnected graph which has vertices of even degree only and contains no Euler circuit. Explain how you know that your example contains no Euler circuit.

9. Comment on Euler's proof of this theorem and corollary as they appear in paragraphs 16 - 19. How convincing do you find his proof? Where and how does he make use of the assumption that the graph is connected in his proof?

1000000000

The various excerpts we have examined from this project demonstrate another emphasized pedagogical goal: that of providing practice moving from verbal descriptions to precise mathematical formulations (Goal 2). In fact, Euler's entire paper provides students with a model for this, as he moves from a detailed map of Königsberg (Euler's Fig. 1.1), to a simpler diagram showing only the main features of the problem (Euler's Fig. 1.2), to a reformulation of the problem in terms of sequences of letters (vertices), to his first algorithm for determining if a solution exists, to the final theorem with which he concludes his paper. Again, project questions interspersed between the source excerpts in which Euler makes these moves provide students with opportunities to reflect upon these reformulations, and apply them in specific cases.

Finally, we note that a deliberate effort was made while designing this project to include questions focused on developing students' verbal and deductive skills through reading (Goal 1), by prompting them to interact with Euler's text in the way which a mature mathematical reader naturally approaches a new text. For example, returning to an earlier part of Euler's paper in which he is developing a procedure for determining how many times a vertex must appear in the representation of a route for a given bridge problem, the following project question prompts students to more deeply reflect on the passage they have just read by applying Euler's rule to a particular 
example; they are then able to immediately compare their conclusion concerning that example to Euler's own conclusion in the subsequent paragraph of his paper.

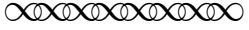

3. In paragraph 8, Euler deduces a rule for determining how many times a vertex must appear in the representation of the route for a given bridge problem for the case where an odd number of bridges leads to the land mass represented by that vertex. Before reading further, use this rule to determine how many times each of the vertices A, B , C and D would appear in the representation of a route for the Königsberg Bridge Problem. Given Euler's earlier conclusion (paragraph 5) that a solution to this problem requires a sequence of 8 vertices, is such a sequence possible? Explain.

10000000000

By incorporating such a diversity of question types - some aimed at developing students' skills in reading and proof writing, others aimed at comprehension of Euler's analysis and its relation to modern graph theory, and still others aimed at promoting reflection on present-day proof standards - this and other projects in the collection naturally lend themselves to the use of multiple approaches to their implementation which can be of particular benefit to students with different learning styles (Goal 8). For instance, the first part of this particular project (in which students are required to read and understand Euler's analysis of the problem) is well suited for small group and whole class discussion, while the final exercises (in which students complete a modern proof of Euler's main theorem) are ideally suited for individual practice in proof writing, but could also be completed in small groups. In the next section, we consider other issues related to classroom implementation of the projects. 


\section{Implementation}

Time spent working on the project is time for explanation, exploration, and discovery, for both the instructor and the student. Instructors are encouraged to adapt a project to their particular course by adding or rephrasing some questions, or deleting others to reflect what is actually being covered. ${ }^{5}$ Each project also has guiding notes for the instructor on its use in teaching.

Our own experience with implementation suggests that it is important to be familiar with all details of a project before implementing it with students. We have also found it important to begin early in the course with a discussion of the relevance of the historical piece, its relation to the course curriculum, and how modern textbook techniques owe their development to problems often posed centuries earlier. A comparison with modern techniques could begin as soon as the students have read the related historical passages, or be postponed until after the project is completed.

For use in the classroom, instructors should allow one to several weeks per project, depending on the project(s) selected. Some projects also work well when implemented in several parts spread over the course of the academic term. For certain course topics, the project can simply replace other course activities for a time, with the main course topics learned directly through the project. Each assigned project should count for a significant portion of the course grade (about 20\%) and may take the place of an in-class examination, or be assigned in pieces as homework.

While a project is being implemented, several class activities are possible. Students could be encouraged to work on the project in class, either individually or in small groups, as the instructor monitors and assists their progress and explores meaning in language from time past. Many instructors also assign select project questions for students to complete based on their own reading, prior to a discussion in class. Whole class discussions or brief lectures may also be appropriate at certain junctures.

Some type of student writing or presentation is recommended in conjunction with an historical project. Again, instructors have considerable flexibility in terms of how this is done. One option is to assign and collect written responses to project questions in installments, either before or after class

\footnotetext{
${ }^{5}$ The source files for projects developed under the pilot grant can be downloaded and edited from the web resource (Bezhanishvili et al 2003). Source files for new projects being developed under the expansion grant can be obtained by contacting their authors; contact information is located on the web resource (Barnett et al 2008).
} 
work. Other instructors elect to assign a final detailed paper based on the project, perhaps asking students to compare how the topic is treated in the primary source to how it is treated in a current textbook. Another possibility is to require that each student complete an historical project related to the course curriculum on an independent basis, either individually or in groups, and report on their topic to the class in an oral presentation.

\section{Conclusion}

Evaluation data from the initial pilot study based on student performance in later course work suggests our approach to using history to teach mathematics is effective in promoting students' understanding of the present-day paradigm of the subject. Of course, there are other factors at play that could explain these data, including differing entering preparation for varying groups of students in different courses and semesters, and individual instructor experience and pedagogical style. Efforts to compensate for these confounding variables are part of the more extensive evaluation now underway with our expansion grant.

Following completion of a course using historical projects, students' own perceptions of the benefits of learning from primary sources echo our pedagogical goals. We close with a selection of their comments, allowing students to have the final word. "See how the concepts developed and understand the process." "It ties in better, links can be made." "Appropriate question building." "Helps with English-math conversion." "Gives meaning to problems." "You get an understanding of why you are doing something." "You understand it without the middle man." "It leads me to my own discoveries." "We learn from the best."

\section{ACKNOWLEDGMENT}

Development of the materials described here has been partially supported by the National Science Foundation's Course, Curriculum and Laboratory Improvement Program, Grants DUE-0231113, DUE-0715392 and DUE-0717752. All opinions, findings and conclusions are those of the authors and do not necessarily reflect the views of the NSF.

\section{REFERENCES}

-Barnett, J., Bezhanishvili, G., Leung, H., Lodder, J., Pengelley, D., Ranjan, D., 2009, "Historical Projects in Discrete Mathematics and Computer Science", 
in Resources for Teaching Discrete Mathematics, B. Hopkins (ed.), Washington, D.C.: Mathematical Association of America, MAA Notes volume 74.

-Barnett, J., Bezhanishvili, G., Leung, H., Lodder, J., Pengelley, D., Pivkina, I., Ranjan, D., 2008, Learning Discrete Mathematics and Computer Science via Primary Historical Sources, http://www.cs.nmsu.edu/historical-projects/.

-Bezhanishvili, G., Leung, H., Lodder, J., Pengelley, D., Ranjan, D., 2003, Teaching Discrete Mathematics via Primary Historical Sources, http://www.math.nmsu.edu/hist_projects/.

-Biggs, N., Lloyd, E., Wilson, R., 1976, Graph Theory: 1736-1936, Oxford: Clarendon Press.

-Euler, L., 1758-59, "Solutio Problematis ad Geometriam Situs Pertinentis", Novi Commentarii Academiae Scientarium Imperialis Petropolitanque 7, 9-28.

-Fauvel, J., van Maanen, J., 2000, History in mathematics education, Dordrecht; Boston: Kluwer Academic Publishers.

-Katz, V., 1998, A History of Mathematics: An Introduction, Second Edition, New York: Addison-Wesley.

-Pascal, B., 1991, "Treatise on the Arithmetical Triangle," in Great Books of the Western World, Mortimer Adler (ed.), Chicago: Encyclopædia Britannica, Inc. 